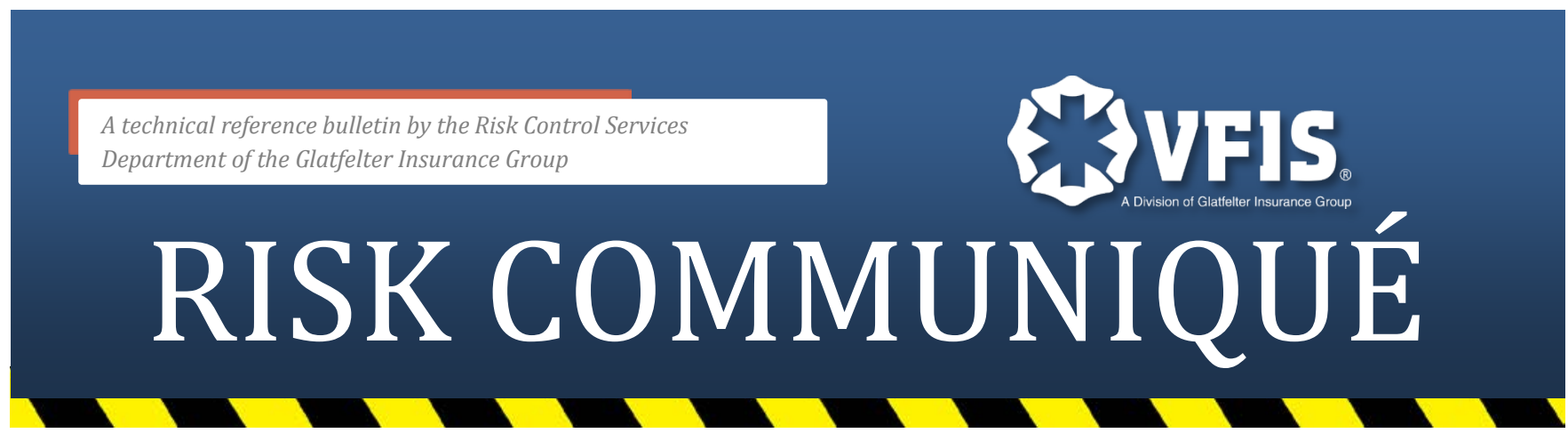

\title{
Intersection Practices
}

Intersections are the locations responsible for the largest percentage of major accidents involving emergency vehicles. Even with the use of warning devices, intersections pose a serious threat to the safety of both emergency service personnel as well as the general public. It's important for emergency service organization to adopt and practice intersection operating guidelines during emergency responses. Review the following best practices and compare them to the organization's SOGs, state statutes and applicable standards. Prior to implementation, have the SOGs reviewed by legal counsel.

\section{Controlled Intersections}

Any intersection controlled by a stop sign, yield sign, yellow traffic light or a red traffic light requires Prudent Action by the emergency vehicle driver. Consider the following steps:

- Do not rely on warning devices to clear traffic

- Scan the intersection for possible hazards (right turns on red, pedestrians, vehicles traveling fast, etc.) and driver options

- Begin to slow down well before reaching the intersection and cover the brake pedal with the drivers' foot, continue to scan in four directions (left, right, front, back)

- When approaching a traffic signal, be aware of "stale" green lights or yellow caution lights, begin to slow and prepare to stop as these will be turning red

- Change the siren cadence not less than $200^{\prime}$ from intersection

- Scan intersection for possible passing options (pass on right, left, wait, etc.) avoid using the opposing lane of traffic if at all possible. Consider using the lane of least resistance that is consistent with your intended direction of travel

- During emergency response, bring the vehicle to a complete stop for any of the following ${ }^{1}$ :

- When directed by a law enforcement officer

- $\quad$ Red traffic lights

- $\quad$ Stop signs

- Negative right-of-way intersection

- When the driver cannot account for visible traffic in the lanes of traffic in an intersection

- When other intersection hazards are present

- When encountering a stopped school bus with flashing warning lights

- Establish eye contact with other vehicle drivers; have partner communicate all is clear; reconfirm all visible traffic is stopped

- Account for traffic one lane at a time, treating each lane of traffic as a separate intersection

- Any time an emergency vehicle driver approaches an unguarded rail crossing, bring the apparatus or vehicle being operated to a complete stop before entering the grade crossing. In addition, have the emergency vehicle driver perform the following prior to proceeding:

- Turn off all sirens and air horns

- $\quad$ Operate the motor at idle speed

This is a sample guideline furnished to you by VFIS. Your organization should review this guideline and make the necessary modifications to meet your organization's needs. The intent of this guideline is to assist you in reducing exposure to the risk of injury, harm, or damage to personnel, property, and the general public. For additional information on this topic, contact your VFIS Risk Control Representative at (800) 233-1957. 


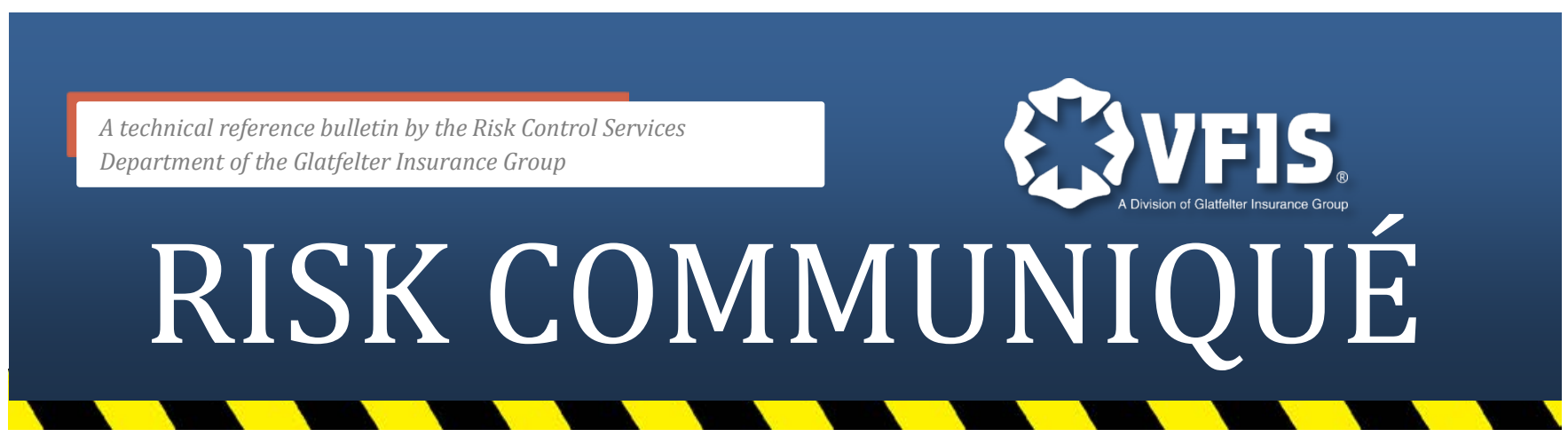

- Turn off any other sound producing equipment or accessories

- Open the windows and listen for a train's horn

\section{Uncontrolled Intersections}

Any intersection that does not offer a control device (stop sign, yield or traffic signal) in the direction of travel of the emergency vehicle, complete the following:

- Scan the intersection for possible hazards (right turns on red, pedestrians, vehicles traveling fast, etc.). Observe traffic in all four directions (left, right, front, rear)

- Slow down if any potential hazards are detected and cover the brake pedal with the driver's foot

- Change the siren cadence not less than $200^{\prime}$ from intersection

- Avoid using the opposing lane of traffic if at all possible

It's important for emergency vehicle drivers to be prepared to slow or bring their vehicle to a stop. If another vehicle operator fails to yield the right of way to an emergency vehicle, the emergency vehicle driver cannot force the right of way, nor can they assume the right of way, therefore the emergency driver does not have the right of way until the other vehicle yields to them.

${ }^{1}$ National Fire Protection Association: NFPA 1500: Standard on Fire Department Occupational Safety and Health Program; 2013 Edition.

\section{Additional Resources}

A Research Study of Ambulance Operations and Best Practice Considerations for Emergency Medical Services Personnel. Boon, C.M., Avery, L.W. \& Malone, T.B. (2014). dhs.gov

This is a sample guideline furnished to you by VFIS. Your organization should review this guideline and make the necessary modifications to meet your organization's needs. The intent of this guideline is to assist you in reducing exposure to the risk of injury, harm, or damage to personnel, property, and the general public. For additional information on this topic, contact your VFIS Risk Control Representative at (800) 233-1957. 